\title{
AVALIAÇÃO FÍSICAE QUÍMICA DA POLPA DE MARACUJÁ CONGELADA COMERCIALIZADA NA REGIÃO DE BAURU ${ }^{1}$

\author{
KÁTIA RAIMUNDO ${ }^{2}$, REGIANE STELA MAGRI², \\ ELIANE MARIA RAVASI STÉFANO SIMIONATO², ALOÍSIO COSTA SAMPAIO ${ }^{3}$
}

RESUMO - A indústria de polpa de maracujá tem como objetivo a obtenção de produtos com características sensoriais e nutricionais próximas da fruta in natura. O objetivo deste trabalho foi avaliar as informações nutricionais das embalagens e as características físico-químicas das polpas de maracujá congeladas. Foram analisadas 25 amostras, de 07 marcas diferentes, adquiridas em supermercados de Bauru-SP, e região. Apenas uma das marcas analisadas (14,3\%) encontra-se em acordo com a legislação vigente, sendo que as demais apresentam tabelas ultrapassadas ou incompletas. As características físico-químicas diferem entre as marcas comercializadas, principalmente quanto ao teor de ácido ascórbico, sendo que 64,0\% das amostras se encontram em desarcordo com o Regulamento Técnico para Fixação dos Padrões de Identidade e Qualidade (PIQ) para polpa de maracujá do Ministério da Agricultura. As variações observadas entre a polpa in natura e a congelada são menores nos itens: densidade, $\mathrm{pH}$, sólidos solúveis totais, acidez em ácido cítrico e ratio, mas altas quanto ao teor de ácido ascórbico.

Termos para indexação: polpa de maracujá congelada; avaliação físico-química; informação nutricional.

\section{PHYSICAL-CHEMICAL EVALUATION OF THE FROZEN PASSION FRUIT PULP TRADED IN THE BAURU REGION}

\begin{abstract}
The purpose of passion fruit pulp industry is to keep the taste and nutritional characteristics as close as possible to the natural fruit. The objective of this work was to evaluate the packing nutritional information and the physical-chemical characteristics of the frozen passion fruit pulp. It was analyzed 25 pulp samples of the 07 different brand names found in Bauru and nearby cities in the State of São Paulo. Just one of the analyzed brand names (14.3\%) was according to the current Brazilian Food Legislation, the others had exceeded or incomplete tables. The variations of the physical-chemical characteristics for the traded brands were mainly due to the rate of ascorbic acid (vitamin C) and 64\% of the samples also don't follow the rules of the Technical Regulation for Identity and Quality Standards (PIQ) for passion fruit pulp of the Agriculture Ministry. The observed variations between "in natura" and frozen pulp are small for the following items: density, $\mathrm{pH}$, Total Soluble Solid (TSS), acidity in citric acid and ratio, but are high for the rate of ascorbic acid.
\end{abstract}

Index Terms: frozen passion fruit pulp; physical-chemical evaluation; nutritional information.

\section{INTRODUÇÃO}

O maracujazeiro-amarelo (Passiflora edulis Sims f. flavicarpa Deg) é uma planta trepadeira da família das Passifloráceas, originária da América Tropical, sendo largamente cultivada e processada em todo o mundo. A polpa é formada por sementes pretas, cobertas de uma substância amarela e translúcida, ligeiramente ácida e de aroma acentuado, sendo consumida ao natural ou em sucos, sorvetes e doces (De Marchi et al., 2000). Esta cultivar representa cerca de $97 \%$ do volume comercializado em todo o País, estimando-se que mais de $60 \%$ da produção de maracujá-amarelo seja destinada ao consumo in natura (Rossi, 2005).

O Brasil é um dos maiores produtores e exportadores de maracujá, pois a fruta encontra condições favoráveis de desenvolvimento em regiões tropicais e subtropicais, por apresentar diversificada aptidão edafoclimática, maior rendimento de suco, maior acidez e maior produção por hectare (Pio et al., 2003). De acordo com o Agrianual (2008), o Brasil é o maior produtor e consumidor mundial de maracujá, possuindo 35.820

\footnotetext{
'(Trabalho 140-08). Recebido em: 06-06-2008. Aceito para publicação em: 27-03-2009.

${ }^{2}$ Universidade do Sagrado Coração, Centro de Ciências da Saúde - USC - Bauru - Rua Irmã Arminda 10-50, Bauru - SP, CEP 17011-160 - Fone: 2107-7258. Fax: 2107-7000.katia_raimundo@itelefonica.com.br; re_magri2@hotmail.com; esimionato@usc.br

${ }^{3}$ Departamento de Ciências Biológicas - UNESP - Bauru - SP - Brasil. aloisio@fc.unesp.br
} 
ha de área colhida em 2005.

A conservação de frutas na forma de sucos, polpas e outros produtos foi desenvolvida para aumentar o oferecimento das mesmas e para a utilização dos excedentes de produção. A polpa de fruta congelada é o produto obtido da parte comestível da fruta, após trituração e/ou despolpamento e preservação por congelamento. Sua utilização é quase sempre como matéria-prima para processamento de outros produtos, como néctares, sucos, geleias, sorvetes e doces. Geralmente, as polpas são comercializadas em embalagens flexíveis (sacos plásticos de polietileno) ou em embalagens cartonadas assépticas devido à facilidade de manuseio e à proteção contra oxidações. As embalagens, além de evitarem as alterações das características sensoriais do produto, devem satisfazer às necessidades de marketing, custo e disponibilidade, entre outros fatores (Brunini et al., 2002).

Para a industrialização, o fruto com excelente flavor e elevado teor de ácidos orgânicos é preferido. A relação entre o teor de Sólidos Solúveis Totais e Acidez Total Titulável (SST/ATT), denominada ratio, é uma das melhores formas de avaliação do sabor de um fruto. Do ponto de vista industrial, o teor elevado de ATT (acidez total titulável) diminui a necessidade de adição de acidificantes e propicia melhoria nutricional, segurança alimentar e qualidade organoléptica (Rocha et al., 2001).

A indústria de polpa de frutas tem como objetivos a obtenção de produtos com características sensoriais e nutricionais próximas da fruta in natura, segurança microbiológica e qualidade, visando não apenas a atender aos padrões exigidos pela legislação brasileira, como também às exigências do consumidor (Amaro et al., 2002).

Além do fator nutricional, a conveniência continua sendo um fator importante para os consumidores. A conveniência, quando atribuída aos alimentos, relaciona-se com a facilidade de estocagem e de preparo para o consumo doméstico. O contínuo crescimento no consumo de frutas, associado às melhorias que estão sendo introduzidas na qualidade dos alimentos, indicam que as polpas congeladas de frutas tropicais devem continuar ganhando mercado. Entretanto, os consumidores estão colocando um novo padrão de conveniência nos alimentos, sendo que a qualidade e o valor nutricional devem ser preservados. Em decorrência da alta instabilidade das vitaminas e pró-vitaminas, o processamento e a estocagem das frutas podem alterar significativamente a composição qualitativa e quantitativa destes nutrientes (Costa \& Vieira, 2003).
Nota-se a necessidade de avaliar as características físico-químicas presentes na polpa de maracujá congelada, pois vários estudos realizados com polpas de diversos frutos indicam que mais de $50 \%$ destas não se enquadram nos padrões para sucos, conforme a legislação vigente, indicando a urgência na elaboração dos PIQs (Padrões de Identidade e Qualidade) para todos os tipos de polpas, a fim de garantir ao consumidor produtos de qualidade (Oliveira et al. 1999).

Brunini et al. (2002) analisaram as alterações em polpa de manga 'Tommy-Atkins' congeladas e relataram que o conteúdo de vitamina $\mathrm{C}$ diminuiu de $56,11 \mathrm{mg} 100 \mathrm{~g}^{-1}$ iniciais, para $23,72 \mathrm{mg} .100 \mathrm{~g}^{-1} \mathrm{e}$ 16,04mg. $100 \mathrm{~g}^{-1}$ em 20 e 26 semanas, respectivamente, os valores de $\mathrm{pH}$ variaram de 4,04 a 4,38, a acidez total titulável variou, de 0,721 a $0,993 \mathrm{~g}$ de ácido cítrico. $100 \mathrm{~g}^{-1}$, os teores de sólidos solúveis totais variaram de 9,48 a $12,53{ }^{\circ}$ Brix.

Gomes et al. (2006) avaliaram a qualidade físico-química da polpa de maracujá, variedade AFRUVEC, obtida manualmente em laboratório, e os valores obtidos no dia do preparo foram entre 4,13 e 4,64\% de ácido cítrico em 100ml para ATT; 13,72 e 14,88 ${ }^{\circ}$ Brix para SST; 9,78 e $12,67 \mathrm{mg} / 100 \mathrm{ml}$ de vitamina $\mathrm{C}$; 2,51 e 2,69 de $\mathrm{pH}$; 3,03 e 3,51 de ratio e densidade entre 1,041 a 1,053g/ml.

O presente trabalho avaliou as características físico-químicas da polpa de maracujá congelada, comercializada na região de Bauru, com o objetivo de comparar as diferentes marcas existentes neste mercado regional quanto ao cumprimento dos padrões de identidade e Qualidade do Ministério da Agricultura e avaliar a informação nutricional constante nas embalagens.

\section{MATERIAL E MÉTODOS}

Foram analisadas 25 amostras de polpas de maracujá congeladas, de 07 marcas diferentes, adquiridos em supermercados de Bauru-SP, e região, entre os meses de agosto e setembro de 2007. As amostras foram constituídas de 03 unidades de venda de cada marca, por data de fabricação e lote. Foi também verificada a lista de ingredientes, informação nutricional, data de fabricação e validade, sendo verificadas se as mesmas estavam de acordo com a resolução da ANVISA RDC nº 359 e 360 (ANVISA, 2008). Após estas verificações, as polpas foram descongeladas, homogeneizadas e analisadas. As análises foram executadas em triplicata no Laboratório de Análise de Alimentos da Fundação Véritas.

Foram analisados o pH, teores de sólidos 
solúveis totais (SST), acidez total titulável (ATT), ratio, densidade e ácido ascórbico.

A metodologia empregada foi em conformidade com as Normas Analíticas do Instituto Adolfo Lutz (Instituto Adolfo Lutz, 2005), com exceção da determinação do teor de ácido ascórbico determinada pela AOAC modificada (De Marchi et al., 2000).

\section{RESULTADOS E DISCUSSÃO}

Quanto à lista de ingredientes, o único ingrediente presente em todas as embalagens era polpa natural do maracujá, sem conservadores ou demais ingredientes.

Na informação nutricional, apenas uma marca apresentou a tabela nutricional atualizada (amostra A); nas embalagens das outras marcas, a tabela estava incompleta ou não atualizada. A mesma marca apresentava a indicação de possuir vitamina C, para tanto é necessário que a quantidade de vitamina $\mathrm{C}$ na polpa corresponda a, no mínimo, 5\% da Ingestão Diária Recomendada (IDR). Neste caso, a amostra cumpria essa exigência.

Em relação ao prazo de validade, a maioria das marcas apresentava validade de 12 meses, com exceção de uma marca, que possuía validade de 24 meses.

No supermercado, foram encontradas polpas com datas de fabricação entre 4 e 328 dias, o que pode justificar a variação observada nos resultados analíticos, principalmente com respeito ao teor de ácido ascórbico (Tabela 01).

Os valores encontrados de densidade na polpa de maracujá congelada variaram entre 1,034 e $1,060 \mathrm{~g} / \mathrm{mL}$, já na polpa in natura extraída diretamente da fruta em (D+1), foi de 1,047 e 1,053g/mL (Gomes et al., 2006).

Os valores encontrados de $\mathrm{pH}$ na polpa de maracujá congelada variaram de 2,67 a 3,77, valores maiores do que na polpa in natura, extraído diretamente da fruta em $(\mathrm{D}+1)$, realizada no mesmo laboratório, que foram de 2,54 e 2,58 (Gomes et al., 2006). Sendo assim, apenas a marca D com 2 amostras (8\%) não se enquadrou no valor mínimo exigido pelo Regulamento Técnico para Fixação dos Padrões de Identidade e Qualidade (PIQ) para polpa de Maracujá do Ministério da Agricultura (MA, 2007), que estabelece o valor mínimo de 2,70 e valor máximo de 3,80.

Os valores encontrados para sólidos solúveis totais (SST) na polpa de maracujá congelada foram de 9,03 a $13,10^{\circ}$ Brix, já na polpa in natura, extraída diretamente da fruta em (D+1), foi de 14,17 e
14,56 ${ }^{\circ}$ Brix (Gomes et al., 2006); sendo assim, 11 amostras (44\%) não estavam em acordo com o valor mínimo exigido pelo PIQ para polpa de maracujá do Ministério da Agricultura, que estabelece o valor mínimo de $11,0{ }^{\circ}$ Brix, indicando presença de água no produto.

Os valores encontrados para acidez em ácido cítrico, na polpa de maracujá congelada, foram entre 2,14 e 3,81\%, já na polpa in natura, extraída diretamente da fruta, a variação foi de 4,54 e 4,61\% (Gomes et al., 2006); sendo assim, apenas três amostras (12\%) não se enquadraram no valor mínimo exigido pelo PIQ para polpa de maracujá do Ministério da Agricultura, que estabelece o valor mínimo de $2,50 \%$.

Os valores calculados para o ratio na polpa de maracujá congelada foram entre 3,07 e 4,40, já na polpa in natura, extraída diretamente da fruta, foi de 3,13 e 3,18 (Gomes et al., 2006).

O ácido ascórbico é a vitamina que se degrada mais facilmente, comparando-se com outras vitaminas. Ela é estável apenas em meio ácido, na ausência de luz, oxigênio e calor, sendo que os fatores que favorecem a sua degradação são os meios alcalinos, oxigênio, calor, ação da luz, metais, como Fe, Cu e Zn, e a enzima oxidase do ácido ascórbico (Oliveira et al., 1999). Muitas pessoas compram a polpa industrializada pela sua facilidade de consumo e também pelas vitaminas presentes.

Nestas amostras, ocorreu uma grande variação do teor de ácido ascórbico na polpa congelada de diferentes marcas, sendo o valor médio encontrado de 3,61mg/100g, o mínimo 0mg/100g (zero) e o máximo 13,19mg/100g (Tabela 01), já na polpa in natura, extraída diretamente da fruta, estes teores variaram entre de 11,28 e 11,79mg/100g (Gomes et al., 2006), muito acima dos valores observados para as polpas congeladas, mostrando que houve degradação durante o processamento ou a comercialização.

Alguns pesquisadores discordam deste fato, pois, analisando o teor de ácido ascórbico do suco de limão Tahiti natural, adoçado e congelado, não encontraram diferença significativa entre as amostras, e citam que “Ácido ascórbico geralmente é estável em sucos e polpas de frutas congeladas” (Pedrão et al., 1999).

Outra observação seria quanto aos dias de fabricação em que a amostra estaria no momento da análise. Neste ponto, nota-se que, de uma mesma amostra, têm-se resultados em 44 e 226 dias de armazenamento com teores de 2,08 e 3,22mg/100g, respectivamente, e outra com 328 dias apresentou, $0,12 \mathrm{mg} / 100 \mathrm{~g}$. Ainda tem-se outra marca com duas 
amostras analisadas que apresentaram, com 4 dias de armazenamento, 2,84 e 2,93mg/100g, respectivamente.

A perda do teor de ácido ascórbico pode ser atribuída às diferenças de qualidade das matériasprimas que são adquiridas de diversas fontes, como produção própria, Centrais de Abastecimento, outros Estados, ou ainda devido às condições de processamento inadequadas (Oliveira et al., 1999). Grande parte das perdas pode ocorrer devido ao tempo gasto para o congelamento total das polpas, podendo ter uma variação entre as marcas, dia do congelamento e supermercados (Ciabotti et al., 2000). Outra hipótese é em decorrência da incorporação de ar durante as etapas de processamento, que favorecem as reações aeróbicas de degradação, bem como a temperatura de armazenamento (Lima et al., 2000). Também podem ocorrer perdas devido aos diferentes tipos de equipamentos utilizados durante o processo e/ou pela oxidação química do ácido ascórbico e/ou degradação térmica através do branqueamento, cozimento, pasteurização e desidratação (Maia et al., 2007).

TABELA 01 - Resultados das análises físico-químicas das polpas de maracujá congeladas.

\begin{tabular}{|c|c|c|c|c|c|c|c|c|c|c|c|}
\hline \multirow[t]{2}{*}{ Marcas } & \multirow[t]{2}{*}{$\begin{array}{l}(\mathrm{D}+\mathrm{n})^{*} \\
\text { Amostras }\end{array}$} & \multirow[t]{2}{*}{$\begin{array}{c}\text { Densidade } \\
\left(\mathrm{g} \cdot \mathrm{mL}^{-1}\right)\end{array}$} & \multirow[t]{2}{*}{$\begin{array}{c}\mathrm{pH} \\
\text { (média) }\end{array}$} & \multicolumn{2}{|c|}{$\begin{array}{l}\text { SST ** } \\
\left({ }^{\circ} \text { Brix) }\right.\end{array}$} & \multicolumn{2}{|c|}{$\begin{array}{c}\text { Acidez em ácido } \\
\text { cítrico (\%) }\end{array}$} & \multicolumn{2}{|c|}{ Ratio } & \multicolumn{2}{|c|}{$\begin{array}{c}\text { Ácido ascórbico } \\
(\mathrm{mg} / \mathbf{1 0 0 g})\end{array}$} \\
\hline & & & & $\bar{x}$ & $\mathrm{~s}$ & $\bar{\chi}$ & $\mathrm{s}$ & $\bar{\chi}$ & $\mathrm{s}$ & $\bar{\chi}$ & $\mathrm{s}$ \\
\hline \multirow{7}{*}{$\mathbf{A}$} & 36 & 1,047 & 2,73 & 12,47 & 0,28 & 3,45 & zero & 3,62 & 0,06 & 13,19 & 0,13 \\
\hline & 50 & 1,049 & 3,03 & 11,97 & 0,29 & 3,36 & zero & 3,57 & 0,17 & 10,3 & 0,24 \\
\hline & 51 & 1,048 & 2,92 & 12,16 & 0,76 & 3,49 & zero & 3,49 & 0,22 & 11,05 & 0,14 \\
\hline & 55 & 1,042 & 3,57 & 13,1 & zero & 2,27 & zero & 4 & 0,04 & 6,06 & 0,47 \\
\hline & 76 & 1,041 & 2,75 & 12,13 & 0,29 & 3,31 & zero & 3,67 & 0,09 & 10,87 & 0,73 \\
\hline & 158 & 1,052 & 3,57 & 12,65 & 0,02 & 3,49 & zero & 3,63 & 0,06 & 5,62 & 0,07 \\
\hline & ------ & 1,043 & 3,1 & 11,97 & 0,29 & 3,44 & zero & 3,49 & 0,13 & 11,66 & 1,65 \\
\hline \multirow{3}{*}{ B } & 140 & 1,038 & 3,06 & 9,03 & 0,5 & 2,38 & zero & 3,79 & 0,09 & 0,43 & 0,07 \\
\hline & 145 & 1,041 & 2,72 & 10,01 & 0,29 & 2,73 & zero & 3,67 & 0,09 & 0,51 & zero \\
\hline & 160 & 1,042 & 3,18 & 9,9 & 0,02 & 2,54 & zero & 3,9 & 0,07 & 0,13 & zero \\
\hline \multirow{3}{*}{$\mathrm{C}$} & 209 & 1,042 & 3,12 & 9,4 & 0,02 & 2,14 & zero & 4,4 & 0,21 & 0,13 & zero \\
\hline & 215 & 1,039 & 3,77 & 10,32 & 0,02 & 2,64 & zero & 3,91 & 0,1 & 0,04 & 0,07 \\
\hline & 4 & 1,035 & 2,67 & 9,55 & 0,29 & 3,04 & zero & 3,14 & 0,12 & 2,93 & 0,12 \\
\hline \multirow[t]{2}{*}{ D } & 4 & 1,034 & 2,67 & 9,22 & zero & 3 & zero & 3,07 & 0,06 & 2,84 & 0,27 \\
\hline & 51 & 1,048 & 2,74 & 12,13 & 0,76 & 3,36 & zero & 3,61 & 0,17 & 3,9 & 0,19 \\
\hline \multirow[t]{2}{*}{ E } & 75 & 1,047 & 3,48 & 11,81 & 0,58 & 3,42 & zero & 3,45 & 0,17 & 0,25 & 0,12 \\
\hline & 44 & 1,041 & 2,96 & 11,02 & zero & 2,78 & zero & 3,96 & 0,06 & 2,08 & 0,12 \\
\hline \multirow[t]{2}{*}{$\mathbf{F}$} & 226 & 1,042 & 2,9 & 11,08 & zero & 3,5 & zero & 3,17 & 0,06 & 3,22 & 0,22 \\
\hline & 328 & 1,049 & 2,9 & 11,72 & zero & 3,8 & zero & 3,07 & 0,06 & 0,49 & 0,12 \\
\hline \multirow{6}{*}{ G } & 42 & 1,04 & 3 & 10,53 & 0,02 & 2,84 & zero & 3,67 & 0,04 & 1,67 & 0,25 \\
\hline & 105 & 1,06 & 3,59 & 13,6 & zero & 3,24 & zero & 4,2 & 0,02 & zero & zero \\
\hline & 190 & 1,043 & 3,59 & 11,52 & zero & 2,84 & zero & 4,06 & 0,02 & 0,13 & 0,13 \\
\hline & 202 & 1,041 & 2,78 & 10,16 & 0,29 & 2,6 & zero & 3,9 & 0,05 & 1,17 & 0,13 \\
\hline & 205 & 1,043 & 2,81 & 10,07 & 0,14 & 2,65 & zero & 3,8 & 0,03 & 0,42 & 0,07 \\
\hline & ------ & 1,042 & 3,15 & 10,49 & zero & 2,64 & zero & 3,98 & 0,08 & 1,13 & 0,08 \\
\hline
\end{tabular}

* $(\mathrm{D}+\mathrm{n})$ - número de dias que as polpas apresentavam quando foram analisadas

* *SST - Sólidos Solúveis Totais

*** $\mathrm{s}-$ Desvio-Padrão

$* * * * \bar{X}-$ Média

\section{CONCLUSÕES}

1-Nas informações nutricionais das embalagens das polpas de maracujá congeladas, apenas uma das marcas analisadas (14,3\% das marcas) se encontra em acordo com a resolução da ANVISA RDC n 359 e 360, sendo que as demais apresentam tabelas ultrapassadas ou incompletas.

2-O Regulamento Técnico para a Fixação dos Padrões de Identidade e Qualidade (PIQ) para polpa de Maracujá do Ministério da Agricultura estabelece o valor mínimo $11^{\circ}$ Brix para teor de sólidos solúveis totais (SST), 2,50\% para acidez em ácido cítrico e
2,70 a 3,80 para $\mathrm{pH}$, sendo que $44 \%$ das amostras não atendem ao mínimo de SST, $12 \%$ não estão em acordo com a acidez e 8,0\% não se enquadram na faixa de $\mathrm{pH}$ estabelecida. As irregularidades observadas indicam maior necessidade de ações de fiscalização quanto ao cumprimento das legislações e padrões estabelecidos.

3-As variações observadas entre polpa de maracujá recém-extraída da fruta e a polpa industrializada congelada são menores nos itens: densidade, pH, SST, acidez em ácido cítrico e ratio, mas extremamente elevadas quanto ao teor de ácido ascórbico, confirmando a sensibilidade desta vitamina em relação ao processamento. 


\section{AGRADECIMENTOS}

Ao Laboratório de Análises de Alimentos da Fundação Véritas.

\section{REFERÊNCIAS}

AGRIANUAL 2007: anuário estatístico da agricultura brasileira. São Paulo:FNP Consultoria e Comércio, 2008. p.388.

AMARO, A. P.; BONILHA, P. R. M.; MONTEIRO, $M$. Efeito do tratamento térmico nas características físico-químicas e microbiológicas da polpa de maracujá. Alimentos e Nutrição, São Paulo, v. 13, p. 151-162, 2002.

ANVISA - Agência Nacional de Vigilância Sanitária. Legislação. Disponível em: <http:// www.anvisa.gov.br/legis/index.htm>. Acesso em: 05 fev. 2008.

BRASIL - Ministério da Agricultura. Regulamento técnico para fixação dos padrões de identidade e qualidade para polpa de maracujá. Disponível em: $<$ http://extranet.agricultura.gov.br/sislegis-consulta/ servlet/VisualizarAnexo?id=1617>. Acesso em : 05 set. 2007.

BRUNINI, M. A.; DURIGAN, J. F.; OLIVEIRA, A. L. Avaliação das alterações em polpa de manga 'Tommy-Atkins' congeladas. Revista Brasileira de Fruticultura, Jaboticabal, v. 24, n. 3, p. 651-653, 2002.

CIABOTTI, E. D.; BRAGA, M. E. D.; MATA, M. E. R. M. C. Alterações das características físicoquímicas da polpa de maracujá-amarelo submetido a diferentes técnicas de congelamento inicial. Revista Brasileira de Produtos Agroindustriais, Campina Grande, v. 2, n. 1, p. 51-60, 2000.

COSTA, T. A.; VIEIRA, R. F. Polpa congelada de acerola: conveniência e qualidade nutricional. v. 25, abr. 2003. Disponível em: $<$ www.clubedofazendeiro.com.br>. Acesso em: 18 ago. 2006.

DE MARCHI, R.; MONTEIRO, M.; BENATO, E. A.; SILVA, C. A. R. Uso da cor da casca como indicador de qualidade do maracujá-amarelo (Passiflora edulis Sim. F. flavicarpa Deg.) destinado à industrialização. Ciência e Tecnologia de Alimentos, Campinas, v. 20, n. 3, p. 110-128, 2000.
GOMES, T. S.; CHIBA, H. T.; SIMIONATO, E. M. R. S.; SAMPAIO, A. C. Monitoramento da qualidade da polpa de maracujá-amarelo - seleção AFRUVEC, em função do tempo de armazenamento dos frutos. Revista Alimentos e Nutrição, Bauru, v.17, n.4, p. 401-405, 2006.

INSTITUTO ADOLFO LUTZ. Métodos Físicoquímicos para análise de alimentos. 4. ed. São Paulo, 2005. 1018p.

LIMA, V. L.A. G; MÉLO, E. A.; LIMA, L. S. Avaliação da qualidade de suco de laranja industrializado. Boletim CEPPA, Curitiba, v. 18, n. 1, p. 95-104, 2000.

MAIA, G. A.; SOUZA, P. H. M.; SANTOS, G. M.; SILVA, D. S.; FERNANDES, A. G.; PRADO, G. M. Efeito do processamento sobre componentes do suco de acerola. Ciência e Tecnologia de Alimentos, Campinas, v. 27, n. 1, p. 130-134, jan./mar. 2007.

OLIVEIRA, M. E. B.; BASTOS, M. S. R.; FEITOSA, T.; BRANCO, M. A.A.C.; SILVA, M. G. G. Avaliação de parâmetros de qualidade físico-químicos de polpas congeladas de acerola, cajá e caju. Ciências e Tecnologia de Alimentos, Campinas, v. 19, n. 3, 1999.

PEDRÃO, M. R.; BELEIA, A.; MODESTA, R. C. D.; FERREIRA, S. H. P. Estabilidade físico-química e sensorial do suco de limão Tahiti natural e adoçado, congelado. Ciência e Tecnologia de Alimentos, Campinas, v. 19, n. 2, 1999.

PIO, R.; RAMOS, J. D.; MENDONÇA, V.; GONTIJO, T. C. A.; RUFINI, J. C. M.; JUNQUEIRA, K. P. Caracterização físico-química dos frutos de sete seleções de maracujazeiro-amarelo para a região de Lavras-MG. Revista Ceres, Viçosa, v. 50, n. 291, p. 573-582, 2003.

ROCHA, M. C.; SILVA, A. L. B.; ALMEIDA, A.; COLLAD, F. H. Efeito do uso de biofertilizante agrobio sobre as características físico-químicas na pós-colheita do maracujá-amarelo (Passiflora edulis f. flavicarpa Deg.) no município de Taubaté. Revista Biociências, Taubaté, v. 7, n. 2, p. 7-13, 2001.

ROSSI, A. D. Situação atual da produção de maracujá no Estado de São Paulo. In: SEMINÁRIO SOBRE MANEJO NO CONTROLE DO VÍRUS DO ENDURECIMENTO DOS FRUTOS (PWV) DO MARACUJAZEIRO, 2005, Bauru. Palestras... Jaboticabal: Gráfica Multipress, 2005. p. 9-20. 